\title{
Cooperative Target Convergence Using Multiple Agents
}

\author{
Kwan S. Kwok \\ Brian J. Driessen \\ (Sandia National Laboratories, Albuquerque, NM 87185-1003)
}

RECEIVED

SEP 231997

OSTI

\begin{abstract}
This work considers the problem of causing multiple (100's) autonomous mobile robots to converge to a target and provides a "follow-the-leader" approach to the problem. Each robot has only a limited-range sensor for sensing the target and also larger but also limited-range robot-to-robot communication capability. Because of the small amount of information available to the robots, a practical approach to improve convergence to the target is to have a robot follow the robot with the best quality of information. Specifically, each robot emits a signal that informs in-range robots what its status is. A robot has a status value of 0 if it is itself in range of the target. A robot has a status of 1 if it is not in range of the target but is is communication range of a robot that is in range of the target. A robot has a status of 2 if it is not in range of the target but is within range of another robot that has status 1 , and so on. Of all the mobile robots that any given robot is in range of, it follows the one with the best status. The emergent behavior is the ant-like trails of robots following each other toward the target. If the robot is not in range of another robot that is either in range of the target or following another robot, the robot will assign -1 to its quality-of-information, and will execute an exhaustive search. The exhuastive search will continue until it encounters either the target or another robot with a nonnegative quality-of-information. The quality of information approach was extended to the case where each robot only has two-bit signals informing it of distance to in-range robots.
\end{abstract}

Keywords: cooperative behavior, multiple agent, emergent behavior, fuzzy logic, multiple target

\section{Introduction}

DIGTREUTION OF THS DOCUMERT IS UHAMTED IP

There has been significant recent interest in the use of multiple robotic agents or robotic vehicles to achieve tasks that a single robot could not achieve alone or that could be accomplished much more efficiently or robustly with multiple robotic devices. There are various approaches to controlling multiple robotic devices. One is to have each behave independently or autonomously, but receive information from its neighbors. The 


\section{DISCLAIMER}

This report was prepared as an account of work sponsored by an agency of the United States Government. Neither the United States Government nor any agency thereof, nor any of their employees, makes any warranty, express or implied, or assumes any legal liability or responsibility for the accuracy, completeness, or usefulness of any information, apparatus, product, or process disclosed, or represents that its use would not infringe privately owned rights. Reference herein to any specific commercial product, process, or service by trade name, trademark, manufacturer, or otherwise does not necessarily constitute or imply its endorsement, recommendation, or favoring by the United States Government or any agency thereof. The views and opinions of authors expressed herein do not necessarily state or reflect those of the United States Government or any agency thereof. 


\section{DISCLAMMER}

Portions of this document may be illegible in electronic image products. Iroges are produced from the best available original doenoment 
receipt of such information could be limited to neighbors that are within a certain communication range (for covertness) of the robotic device. The other extreme is to have a centralized base station, either mobile or fixed, that collects position and other information on all of the vehicles and commands them to move to certain locations.

Reynolds [8] considered the formation of flocks, herds, and schools in simulations in which multiple autonomous agents were driven away from each other and other obstacles by inverse square law repulsive forces. Part of the motivation behind Reynold's work was the impression of centralized control exhibited by actual bird flocks, animal herds, and fish schools, despite the fact that each agent (bird, animal, or fish) is responding only to its limited-range local perception of the world, in Reynold's opinion. Reynolds stated "Natural flocks seem to consist of two balanced, opposing behaviors: a desire to stay close to the flock and a desire to avoid collisions within the flock." He also stated "A bird might be aware of three categories: itself, its two or three nearest neighbors, and the rest of the flock." The desire to be close to the flock while avoiding collisions was modeled by force fields that attracted a bird to its two to three closest neighbors but prevented it from getting too close to these neighbors.

Arkin [1] studied an approach to "cooperation without communication," for multiple mobile robots. He states that "by enabling many simple robots to cooperate together on a large task, it becomes possible to solve a problem that would be infeasible using a large-scale machine." Arkin also points out that centralized master/slave or hierarchy-based approaches have drawbacks of potential communication bottlenecks and less robustness than completely decentralized approaches in which each agent is autonomous and has the same control law. Arkin demonstrated such an approach for robots that are supposed to forage and retrieve objects (in a hostile environment). Anticipated applications mentioned were "housekeeping on the exterior of a space station or undersea base" and "mining operations at remote locations." Kube and Zhang [6] also considered decentralized robots performing tasks "without explicit communication." Much of their study examined comparisons of behaviors of different social insects such as ants and bees. They considered a box-pushing task and utilized a subsumption approach as well as ALN (Adaptive Logic Networks). Asama [2] intelligently points out that "an autonomous and decentralized system has two essentially contradictory characteristics, autonomy and cooperativeness, and the biggest problem in the study of distributed autonomous robotic systems is how to reconcile these two features."

Noreils [7] conducted work toward achieving cooperation between mobile robots for indoor environment applications. He dealt with robots that were not necessarily homogeneous. That is, one subset of the robots may have capabilities that another subset does not have. His architecture consisted of three levels: functional level, control level, and planner level. The planner level was the high-level decision maker. He developed a special purpose language for his application. 
Chen and Luh [5] examined decentralized control laws that drove a set of mobile robots into a specific formation. In particular, a circle formation was considered. Similarly, Yamaguchi and Arai [9] studied line-formations, and so did Yoshida et al, [10].

The work contained herein will present a cooperative behavior in which robotic vehicles are able to achieve much better convergence to a target via the ability to have the distance to the target of other vehicles communicated to them. With more information available to it, each vehicle is better at converging to the target.

\section{Problem Statement}

This section describes the problem of interest and section 3 discusses a proposed solution to this problem. We have $\mathrm{N}$ mobile robotic vehicles. Each vehicle has a sensor that informs the vehicle of its distance to the goal or target. Also, each vehicle is able to communicate only with vehicles that are within a certain range $\left(r_{f}\right)$. For such in-range vehicles, sensors are available that inform the vehicle of its distance to these other vehicles, but only to within a resolution of 2 bits: Near, Medium, Far, No Signal. Also, the distance to the target information is also available only to within 2 bits, and a compass bearing to in-range vehicles and the goal (if in range) is available.

Again, each vehicle has 2 bits of distance information, from both the source and to other vehicles. A vehicle can communicate with another vehicle only if it is within distance $r_{f}$ of this vehicle. A vehicle knows only whether it is Near, Medium, or Far distance away from another vehicle. In particular, if we let $r$ be the distance to the other vehicle, then

Near: $\mathrm{r}<r_{n}$

Medium: $r_{n} \leq \mathrm{r} \leq r_{m}$

Far: $r_{m}<\mathrm{r} \leq r_{f}$

No signal: $r>r_{f}$

Likewise for distances $\mathrm{R}$ to the target.

Near: $\mathrm{R}<R_{n}$

Medium: $R_{n} \leq \mathrm{R} \leq R_{m}$

Far: $R_{m}<\mathrm{R} \leq R_{f}$

No Signal: $R>R_{f}$

An example set of values that will be used in the simulation results of section 4 is:

$R_{f}=1000$

$R_{m}=R_{f} / 2$ 
$R_{n}=R_{m} / 2$

$r_{f}=R_{f}^{*} 10$

$r_{m}=r_{f} / 2$

$r_{n}=r_{m} / 2$

(The sensing range to the target typically much less than vehicle-to-vehicle communication range.)

Each vehicle has a compass so that it knows which direction it is pointing (North, South, East, or West, or anything in between). Again, presently each vehicle is assumed to know relative bearing (direction) to vehicles that are within distance $r_{f}$ of itself. Each vehicle has three possible velocities: $v_{\max }, \bar{v}=v_{\max } / 4$, and zero. The objective is to get as many of the vehicles to converge to the target as possible.

\section{Problem Solution Approach}

This section describes a proposed solution to the problem of section 2. The main idea is to try to use the larger vehicle-to-vehicle communication range to overcome the small vehicle-to-target sensing range. One approach is to have a vehicle follow the communication-range vehicle whose status is best. A vehicle has the best possible status (status $=0$ ) if it is within range of the target. A vehicle has second best status (status=1) if it is not within range of the target but is within range of another vehicle with status $=0$, and so on. (That is, of all the vehicles that are within communication range, the vehicle chooses to follow the one that has the best status.) Vehicles that are out of range of the target are drawn into the target by following other vehicles that are in range of the target or by following vehicles that are following vehicles that are within range of the target, etc. Vehicles that are within range of the target simply move toward the target at velocity $v_{\max } / 4$.

If the robot is not in range of another robot that is either in range of the target or following another robot, the robot will assign -1 to its quality-of-information (i.e., its status) and will execute an asterisk-shaped exhaustive search pattern otherwise. The center of this asterisk is intended to be the initial position of the robot and the radius of this asterisk is some assumed bound on the distance the robot could possibly be from the target. If this bound is correct and if the vehicle could (although unrealistically) perfectly control its velocity and compass heading, all vehicles would eventually reach the goal.

Each vehicle is programmed with a few simple rules. The rules used for following another vehicle is to move toward the vehicle being followed, with the following velocity rules (where again $r$ is the distance to the best detectable vehicle to follow). 


$$
\begin{aligned}
& v=v_{\max } \text { if } \mathrm{r}=\text { Far } \\
& \mathrm{v}=\nabla=\mathrm{v}_{\max } / 4 \text { if } \mathrm{r}=\text { Medium } \\
& \mathrm{v}=\mathrm{v}_{\max } / 16 \text { if } \mathrm{r}=\text { Near }
\end{aligned}
$$

Each vehicle assigns itself a status. Statuses are defined as follows:

Status $=-1$ if $\mathrm{R}>R_{f}$ (out of range of the target)

Status $=0$ if $\mathrm{R} \leq R_{f}$ (in range of the target)

Status $=1$ if the vehicle is out of range of the target but within distance $r_{f}$ of a vehicle with status 0 .

Status $=2$ if the vehicle is out of range of the target but within distance $r_{f}$ of a vehicle with status 1 .

Status $=3$ if the vehicle is out of range of the target but within distance $r_{f}$ of a vehicle with status 2 .

and so on. Again, of all the vehicles that a vehicle is within distance $r_{f}$ of, the vehicle chooses to follow the one with the best status. (Status $=0$ is best, status $=1$ is next best, etc., and status $=-1$ is worst.) And the vehicle declares his own status to be 1 more than that of the vehicle it decides to follow. Vehicles that are neither within range of the target nor within range of a vehicle that is range of the target perform the exhaustive asterisk-shape pattern search described above.

\section{Simulation Results}

In this section, simulation results for the problem of section 2 and the solution approach of section 3 are presented, for three initial conditions. These simulation results are in Figures 1, 2, and 3 below. Dots are used to illustrate both the initial and final position of each vehicle. The lines in these figures illustrate the paths taken by the vehicles. Here, in these figures, the emergent ant-like behavior can be discerned through the bundled nature of the spoke-like sets of paths that converge to the target, which was at the origin of the figures' $x-y$ axes.

As a vehicle follows another vehicle who is following another vehicle, who is following another (etc.), the resulting paths start to look like ant-like trails or paths. The approximately three bundles of paths leading to the target in the figures are representative of this type of emergent behavior. We see that some of the vehicles have status -1; that is, they are not in range of the target and have no vehicle to follow. These vehicles are performing the asterisk-shaped exhaustive search. This exhaustive search starts with a move southward followed by a northward move back to its starting position, etc. 


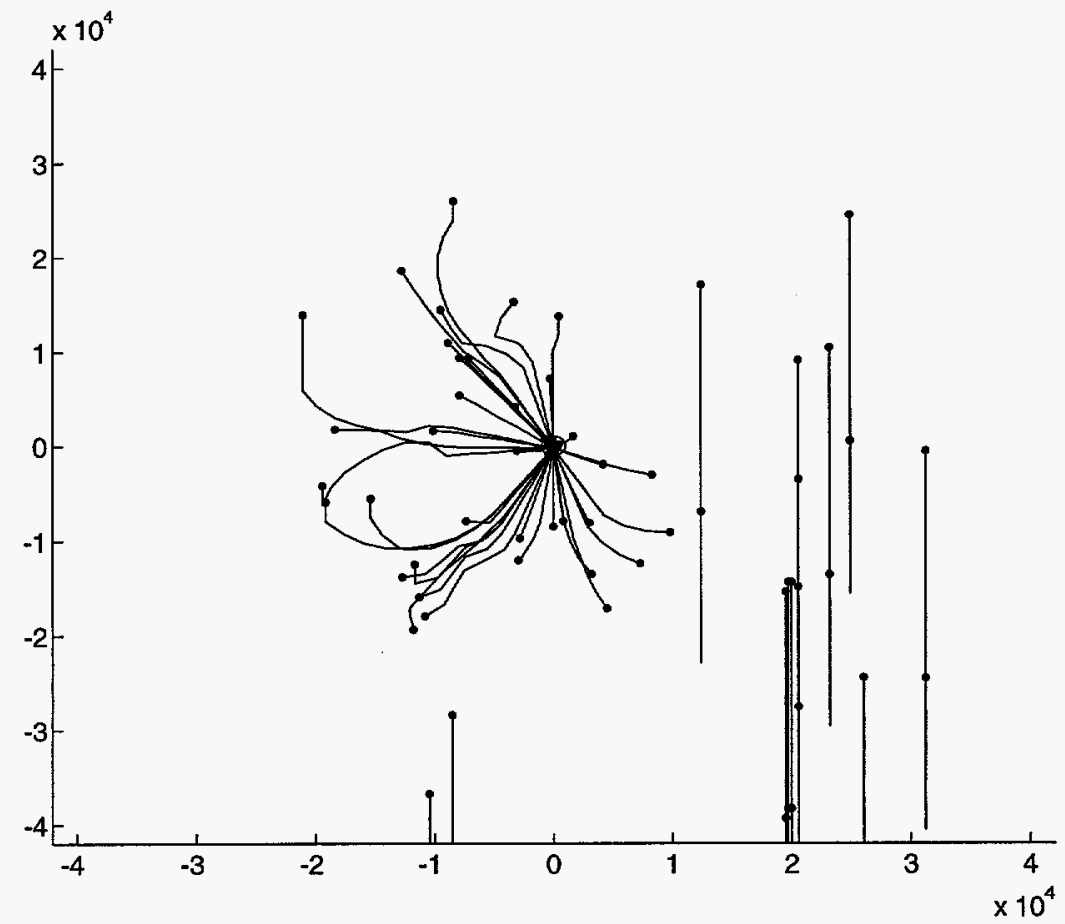

Figure 1. Simulation Results, Case 1

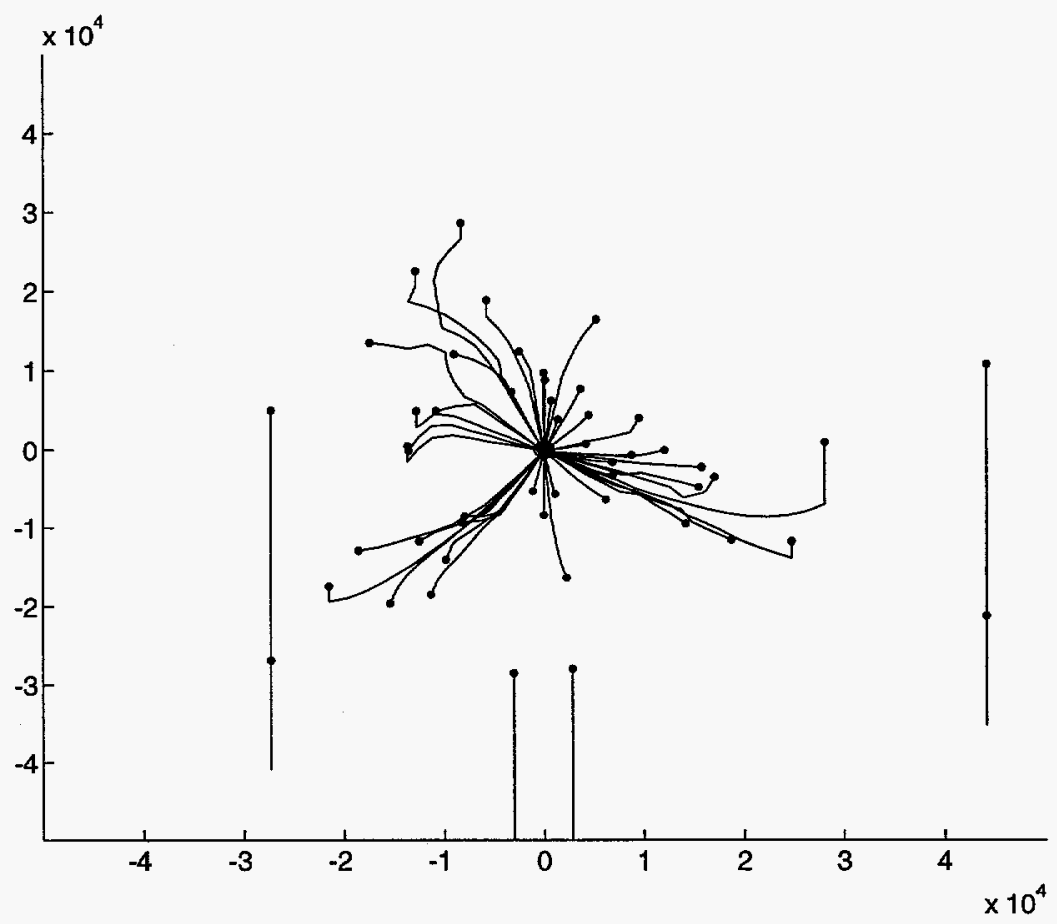

Figure 2. Simulation Results, Case 2 


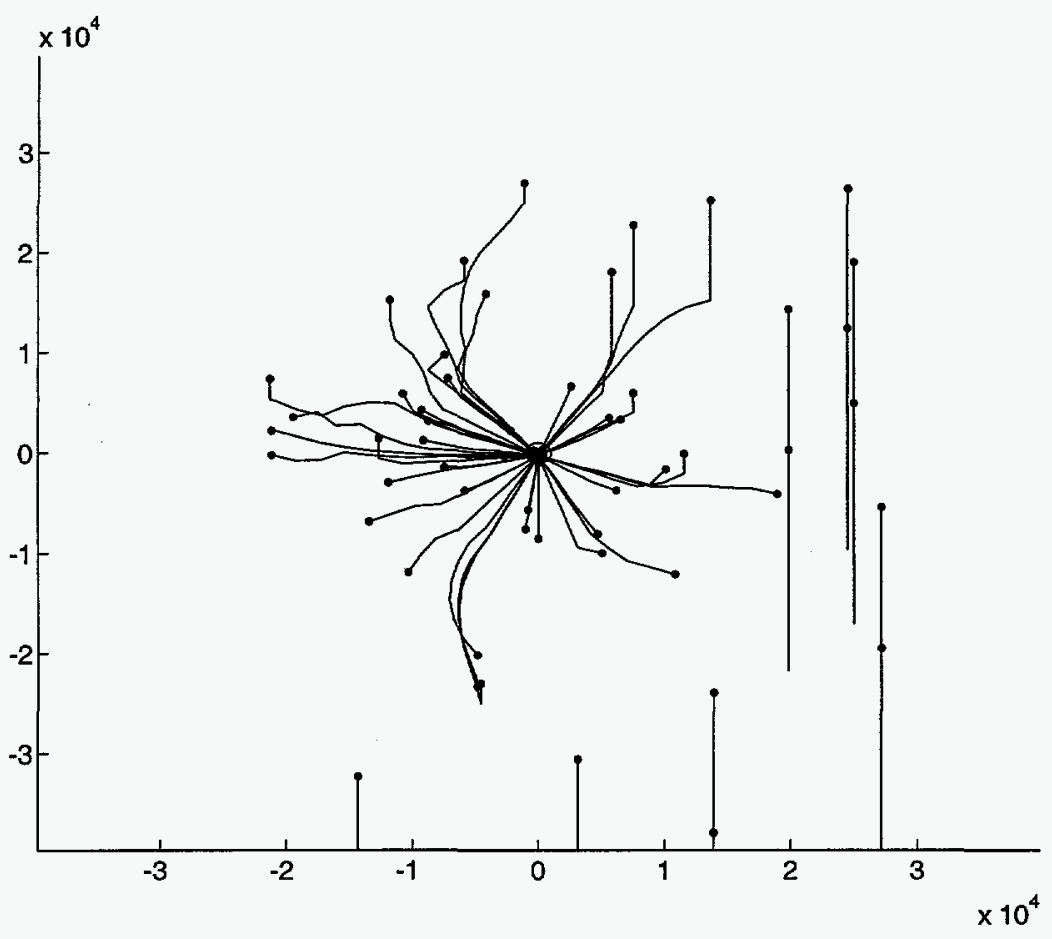

Figure 3. Simulation Results, Case 3

\section{Conclusion}

This work considers the problem of causing multiple (100's) autonomous mobile robots to converge to a target and provides a "follow-the-leader" approach to the problem. Each robot has only a limited-range sensor for sensing the target and largerrange, but also limited, robot-to-robot communication capability. Because of the small amount of information available to the robots, a practical approach to improve convergence to the target is to have a robot follow the robot with the best quality of information. Since the range-to-target sensing capability is small compared to the vehicle-to-vehicle communication range, it makes sense to utilize the longer vehicle-tovehicle communication range in a follow-the-leader type of approach, in which a vehicle follows the communication-range vehicle whose distance to the target is smallest. Specifically, each robot emits a signal that informs in-range robots what its status is. A robot has a status value of 0 if it is itself in range of the target. A robot has a status of 1 if it is not in range of the target but is in range of a vehicle that is in range of the target. A robot has a status of 2 if it is not in range of the target but is within range of another robot that has status 1 , and so on. Of all the mobile robots that any given robot is in range of, it follows the one with the best status. The emergent behavior is the ant-like trails of robots following each other toward the target. Simulation results were presented that illustrate the emergent anti-like behavior. 


\section{ACKNOWLEDGMENTS}

Sandia is a multiprogram laboratory operated by Sandia Corporation, a Lockheed Martin Company, for the United States Department of Energy under Contract DE-AC0494AL85000.

\section{References}

[1] Arkin, Ronald C., "Cooperation Without Communication: Multiagent SchemaBased Robot Navigation," Journal of Robotic Systems 9(3), 1992, pp. 351-364.

[2] Asama, "Distributed Autonomous Robotic System Configured With Multiple Agents and Its Cooperative Behaviors," Journal of Robotics and Mechatronics, Vol. 4, No. 3, 1992, pp. 199-204.

[3] Brooks, Rodney A. and Flynn, Anita M., "Fast, Cheap and Out of Control: A Robot Invasion of the Solar System," Journal of the British Interplanetary Society, Vol. 42, 1989, pp. 478-485.

[4] Brooks, Rodney A., "A Robust Layered Control System for a Mobile Robot," IEEE Journal of Robotics and Automation, Vol. RA-2, No. 1, March 1986, pp. 14-23.

[5] Chen, Qin and Luh, J.Y.S., "Coordination and Control of a Group of Small Mobile Robots," IEEE International Conference on Robotics and Automation, Vol. 3, 1994, pp. 2315-2320.

[6] Kube, Ronald $\mathrm{C}$ and Zhang, Hong, "Collective Robotics: From Social Insects to Robots," Adaptive Behavior, Vol. 2, No.2, pp. 189-218.

[7] Noreils, Fabrice R., "Toward a Robot Architecture Inte grating Cooperation Between Mobile Robots: Application to Indoor Environment," The International Journal of Robotics Research, Vol. 12, No. 1, February 1993, pp. 79-98.

[8] Reynolds, Craig W., "Flocks, Herds, and Schools," Computer Graphics, Volume 21, No. 4, July 1987 , , pp. 25-34.

[9] Yamaguchi, Hiroaki, and Arai, Tamio, "Distributed and Autonomous Control Method for Generating Shape of Multiple Mobile Robot Group," IEEE International Conference on Intelligent Robots and Systems, Vol. 2, 1994, pp.800-807. 
Cooperative Target Convergence Using Multiple Agents

[10] Yoshida, E.; Arai, Tamio, Ota, Jun; Miki, Tomoyoshi, "Effect of Grouping in Local Communication System of Multiple Mobile Robots," IEEE International Conference on Intelligent Robots and Systems, Vol. 2, 1994, pp. 808-815. 